\title{
Evolution of Concepts and Models for Quantifying Resiliency and Flexibility of Chemical Processes
}

\author{
Ignacio E. Grossmann*, Bruno A. Calfa, Pablo Garcia-Herreros \\ Department of Chemical Engineering, Carnegie Mellon University, \\ Pittsburgh, PA 15123, United States
}

Dedicated to Manfred Morari for his pioneering and inspiring research work that has produced major advances in process systems engineering.

Rev. Dec. 2013

\begin{abstract}
This paper provides a historical perspective and an overview of the pioneering work that Manfred Morari developed in the area of resiliency for chemical processes. Motivated by unique counter-intuitive examples, we present a review of the early mathematical formulations and solution methods developed by Grossmann and co-workers for quantifying Static Resiliency (Flexibility). We also give a brief overview of some of the seminal ideas by Manfred Morari and co-workers in the area of Dynamic Resiliency. Finally, we provide a review of some of the recent developments that have taken place since that early work.
\end{abstract}




\section{Introduction}

This paper is a tribute to the pioneering work by Manfred Morari in the area of resiliency that he initiated during his early years at the University of Wisconsin (1977-1983) and later was a major part of his research at Caltech (1983-1994).

We first give an account of the early relation that Ignacio Grossmann was fortunate to establish with Manfred Morari through their discussions on Flexibility and Resiliency, which are major components of the operability of chemical processes. We then provide two motivating examples that show the non-trivial nature of these areas, and which motivated much of the subsequent research. We next give a brief review of the early mathematical formulations and solution methods developed by Grossmann and co-workers for addressing these problems, which can also be found in Biegler et al. (1997). We also briefly review the major contribution by Morari and co-workers. Finally, we close with the new developments and extensions that have taken place since that early work.

\section{The early years of flexibility and resiliency}

Ignacio Grossmann met Manfred Morari in 1980 on their way to Hennicker to attend the $1^{\text {st }}$ FOCAPD meeting organized by the late Dick Mah and Warren Seider. At the time Ignacio asked Manfred: what are you working on these days? He replied: on Resiliency. What about you? Ignacio replied: I am working on Flexibility. They both thought: Flexibility or Resiliency?

According to Merriam Webster, Flexibility is the ready capability to adapt to new, different, or changing requirements, while Resiliency is the capability to recover or adjust easily to misfortune or change. Clearly not much of a difference, except that Flexibility might be better suited for optimists, while Resiliency might be better for pessimists!

After this encounter, Manfred and Ignacio joined forces at the $2^{\text {nd }}$ FOCAPD meeting that took place at Snowmass, and was organized by Art Westerberg and Henry Chien. That led to their joint paper (Grossmann \& Morari, 1984) in which for the first time they quantitatively articulated the properties of Flexibility and Resiliency, with the former generally addressing the capability of feasible operation in the steady state, and the latter addressing the dynamic capability to easily recover from process disturbances in a fast and smooth manner.

More specifically, the motivation of this early work was to incorporate operability considerations at the design stage. The conventional approach is to consider only nominal conditions without anticipating effects of changes and uncertainties in plant operation. The remedy is to use overdesign to compensate for lack of anticipation. In contrast, steady-state Flexibility or Resiliency addresses the guaranteed feasibility of operation of a plant over a range of conditions, with the ultimate goal being on how to design a process for guaranteed flexible/resilient operation. Furthermore, dynamic Resiliency is concerned with fast and smooth changeover and recovery from process disturbance, with the ultimate goal of determining the inherent dynamic characteristic of a plant independent of the selection of a particular controller. To appreciate why these are non-trivial problems we review two motivating examples in the next section. 


\section{Motivating examples}

Let us consider the heat exchanger network shown in Fig. 1 (Biegler et al., 1997), a slight modification of the pioneering example by Saboo and Morari (1984) in which the heat capacity flowrate $F_{H 1}$ is an uncertain parameter. We would like to determine whether this network is feasible for the range $1 \leq F_{H 1} \leq 1.8(\mathrm{~kW} / \mathrm{K})$.

The following inequalities are considered for feasible operation of this network:

Feasibility in exchanger 2:

Feasibility in exchanger 3 :

Feasibility in exchanger 3:

Specification in outlet temperature

$$
\begin{aligned}
& T_{2}-T_{1} \geq 0 \\
& T_{2}-393 \geq 0 \\
& T_{3}-313 \geq 0 \\
& T_{3} \leq 323
\end{aligned}
$$

By considering the corresponding heat balances, we can solve for the above temperatures in terms of the cooling load $Q_{c}$, that can be regarded as a control variable (i.e. degree of freedom), and in terms of $F_{H 1}$, the uncertain parameter. The reduced inequalities in (1) are then as follows:

$$
\begin{aligned}
& f_{1}=-25+Q_{c}\left[\frac{1}{F_{H 1}}-0.5\right]+\frac{10}{F_{H 1}} \leq 0 \\
& f_{2}=-190+\frac{10}{F_{H 1}}+\frac{Q_{c}}{F_{H 1}} \leq 0 \\
& f_{3}=-270+\frac{250}{F_{H 1}}+\frac{Q_{c}}{F_{H 1}} \leq 0 \\
& f_{4}=260-\frac{250}{F_{H 1}}-\frac{Q_{c}}{F_{H 1}} \leq 0
\end{aligned}
$$




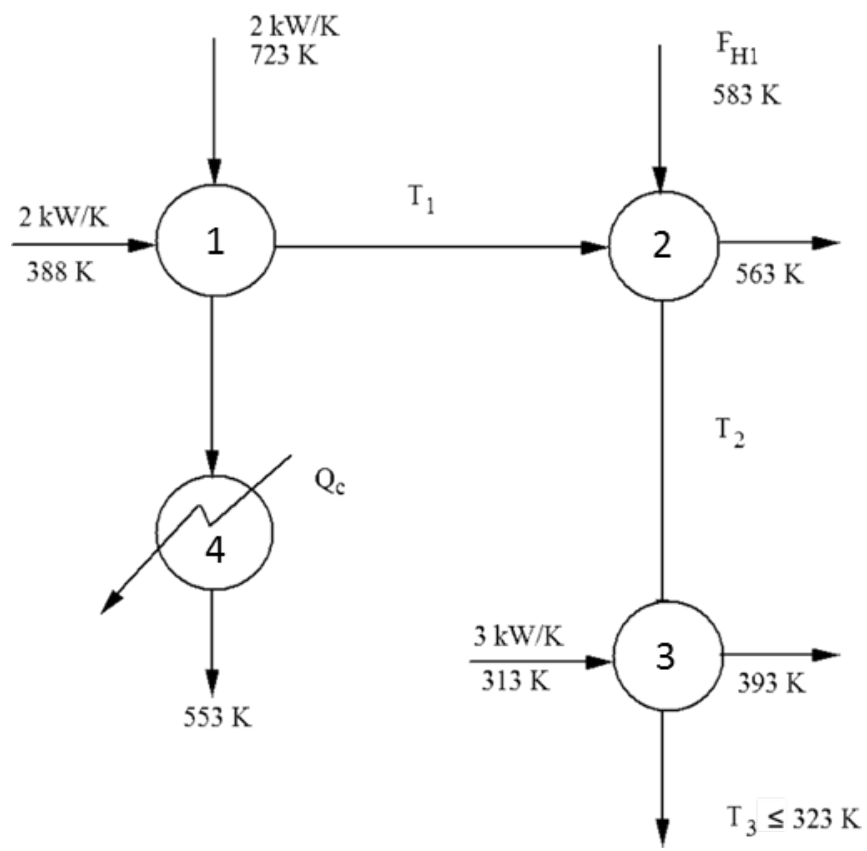

Fig. 1. Heat exchanger network with uncertain heat capacity flowrate, $F_{H 1}$.

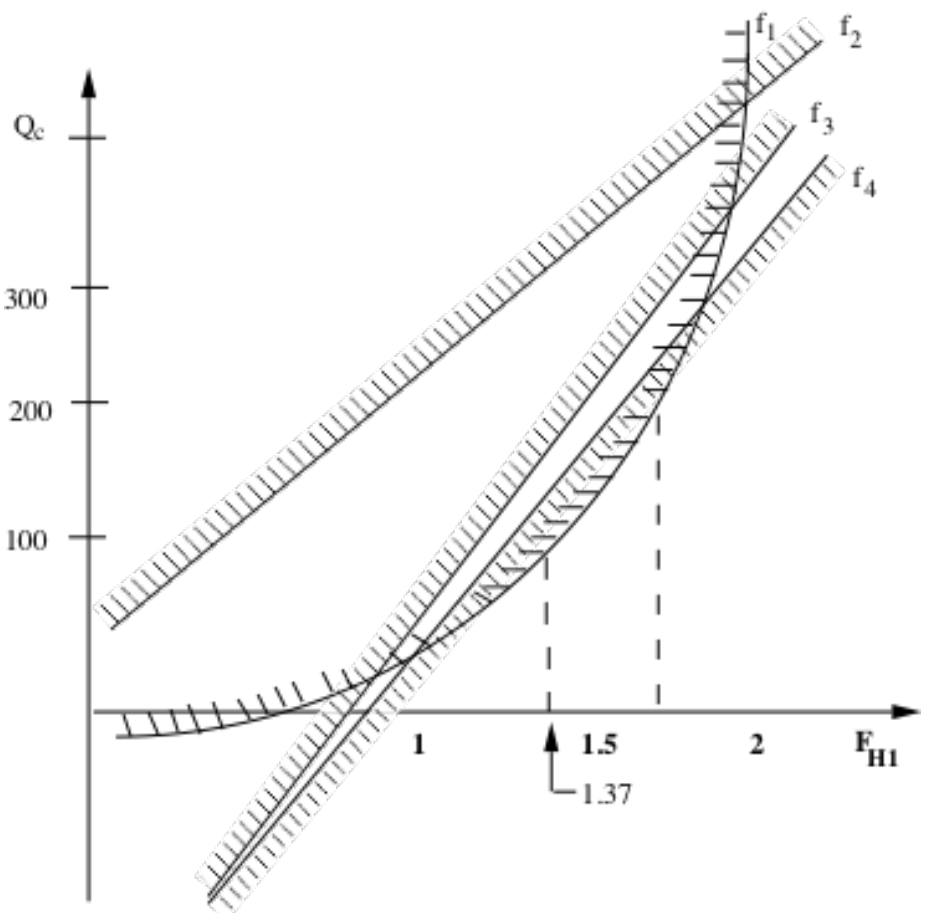

Fig. 2. Feasible region for constraints in (2).

Plotting the inequalities in (2) in terms of $Q_{C}$, the control variable, and in terms of $F_{H 1}$, the uncertain parameter, we can see that the inequalities are satisfied at the extreme points $F_{H 1}=1$ $\mathrm{kW} / \mathrm{K}$, say for $Q_{C}=15 \mathrm{~kW}$, and at $F_{H 1}=1.8 \mathrm{~kW} / \mathrm{K}$, say for $Q_{C}=227 \mathrm{~kW}$. That is, by adjusting the cooling load one can achieve feasibility of operation in the network at the extreme points. If we did not plot the feasible region we may be tempted to conclude that the network is feasible to 
operate for the range $1 \leq F_{H 1} \leq 1.8 \mathrm{~kW} / \mathrm{K}$. However, from Fig. 2, we can see that for an intermediate value, like $F_{H 1}=1.2 \mathrm{~kW} / \mathrm{K}$, the inequalities define an empty feasible space even if we set the cooling load to say $Q_{C}=58.6 \mathrm{~kW}$. In other words the network is infeasible at the nonvertex point $F_{H 1}=1.2 \mathrm{~kW} / \mathrm{K}$. Furthermore, from Fig. 2 we can clearly see that we have a nonconvex region where for $1.118 \leq F_{H 1} \leq 1.65$ we have infeasible operation. In fact at $F_{H 1}=1.37$ $\mathrm{kW} / \mathrm{K}$ we have the greatest violation of constraints. Hence, $F_{H 1}=1.37 \mathrm{~kW} / \mathrm{K}$ corresponds to the critical point. This brilliant example by Saboo \& Morari (1984) shows that it is possible to have non-vertex critical points, and consequently, that we need appropriate methods that will be able to predict such points.

The next motivating example (Grossmann \& Morari, 1984) shows that the sensitivity of a multivariable control system to plant parameter variations is not only a function of the control system design, but even more so of the system itself. Fig. 3 shows a system of thermally coupled distillation columns, which is used to separate a $70 \%$ methanol/water mixture into a $99 \%$ methanol distillate and a $0.1 \%$ methanol bottom product (more details on the model and analyses can be found in Lenhoff \& Morari, 1982).

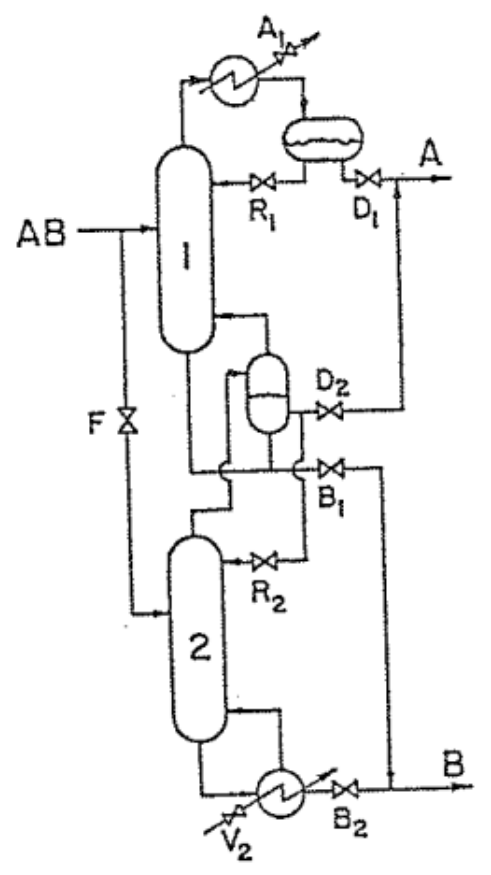

Fig. 3. Thermally coupled distillation columns.

Two control structures are investigated:

$\begin{array}{ccc} & \text { Manipulated Var. } & \text { Fixed Var. } \\ \text { Structure 1 } & R_{1}, V_{2} & F, R_{2} \\ \text { Structure 2 } & F, V_{2} & D_{1}, R_{2}\end{array}$

That is, the manipulated variables are the two valves used for composition control and the fixed variables are not used at all for control. To mimic a situation encountered in a real plant, time 
delays in length equal to about $8 \%$ of the dominant open loop time constants were introduced in the model.

Simulation of the system shows that the performance deteriorates significantly for Structure 1 and much less for Structure 2. Moreover, Structure 1 yields an unstable system for slightly larger time delay or gain error. The contributions by Morari and co-workers provide criteria that can be used to assess the dynamic resilience at the design stage, thus avoiding extensive simulation runs as the differences in sensitivity may not be obvious on physical grounds and may not be suggested by heuristics.

\section{Mathematical formulations for flexibility analysis}

In the previous section we have shown through the motivating example how to graphically perform a flexibility analysis on a simple heat exchanger network. In this section, we will see how we can systematically address these problems through mathematical formulations developed by Grossmann and co-workers (Grossmann et al., 1983; Grossmann \& Straub, 1991). We will then also consider simple vertex solution methods as well as an active set method, which does not necessarily have to examine all the vertex points or even assume that critical points correspond to vertices.

The basic model assumed for the flexibility analysis involves the following vectors of variables and parameters:

$d=$ design variables corresponding to the structure and equipment sizes of the plant

$x=$ state variables that define the system (e.g. flows, temperatures)

$z=$ control variables that can be adjusted during operation (e.g. flows, utility loads)

$\theta=$ uncertain parameters (e.g. inlet conditions, reaction rate constants)

The equations that represent the performance (e.g. heat and material balances) are given by:

$h(d, x, z, \theta)=0$

where by definition $\operatorname{dim}\{h\}=\operatorname{dim}\{x\}$. The constraints that represent feasible operation (e.g. physical constraints, specifications) are given by:

$g(d, x, z, \theta) \leq 0$

Although in principle we can analyze flexibility directly in terms of (3) and (4), for simplicity in the presentation we eliminate the state variables $x$ from (3) as we did in in the motivating example for the heat exchanger network. In this way the state variables become an implicit function of $d, z$, and $\theta$. That is,

$x=x(d, z, \theta)$

Substituting (5) in (4) yields the reduced inequalities

$g(d, x(d, z, \theta), z, \theta)=f(d, z, \theta) \leq 0$ 
Hence, the feasibility of operation of a design $d$ operating at a given value of the uncertain parameters $\theta$ is determined by establishing whether by proper adjustment of the control variables $z$ each inequality $f_{j}(d, z, \theta), j \in J$ is less or equal to zero.

In the next two subsections we present mathematical formulations for both the flexibility test problem and for the flexibility index problem (Halemane \& Grossmann, 1983; Swaney \& Grossmann, 1985a,b).

\subsection{Flexibility test problem}

Assume we are given a nominal value of the uncertain parameters $\theta^{N}$, as well as expected deviations $\Delta \theta^{+}, \Delta \theta^{-}$, in the positive and negative directions. This then implies that the uncertain parameters $\theta$ have the following bounds:

Lower bound: $\theta^{L}=\theta^{N}-\Delta \theta^{-}$

Upper bound: $\theta^{U}=\theta^{N}+\Delta \theta^{+}$

The flexibility test problem (Halemane \& Grossmann, 1983) for a given design $d$ consists in determining whether by proper adjustment of the controls $z$ the inequalities $f_{j}(d, z, \theta), j \in J$, hold for all $\theta \in T=\left\{\theta: \theta^{L} \leq \theta \leq \theta^{U}\right\}$. In order to answer this question, we first consider whether for a fixed value of $\theta$, the controls $z$ can be adjusted to meet the constraints $f_{j}(d, z, \theta)$. This can be accomplished if we select the controls $z$ so as to minimize the largest $f_{j}$, that is,

$\psi(d, \theta)=\min _{z} \max _{j \in J}\left\{f_{j}(d, z, \theta)\right\}$

where $\psi(d, \theta)$ is defined as the feasibility function. If $\psi(d, \theta) \leq 0$, we can have feasible operation; if $\psi(d, \theta)>0$, there is infeasible operation even if we do our best in trying to adjust the control variables $z$. If $\psi(d, \theta)=0$ it also means that we are on the boundary of the region of operation.

Problem (7) can be posed as a standard optimization problem (LP or NLP) by defining a scalar variable $u$, such that:

$\psi(d, \theta)=\min _{z, u} \quad u \quad \quad \forall j \in J$ 


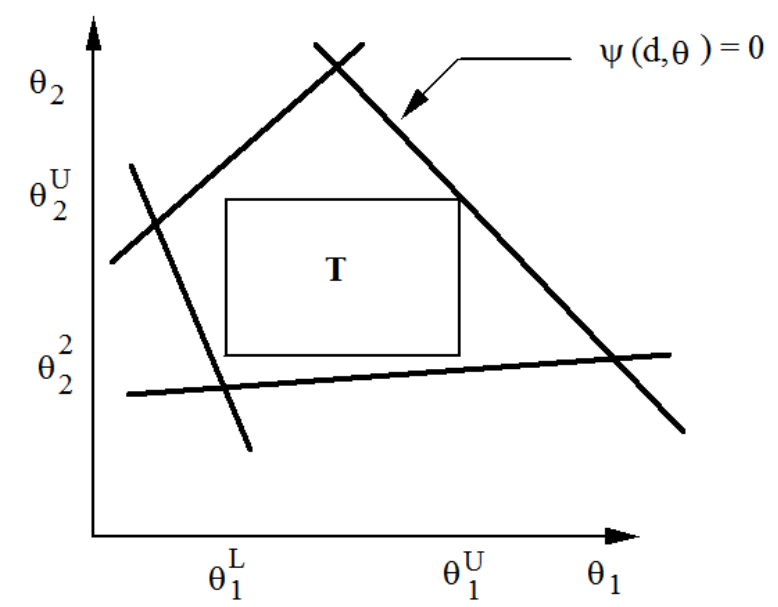

a) Feasible design $\chi(d) \leq 0$

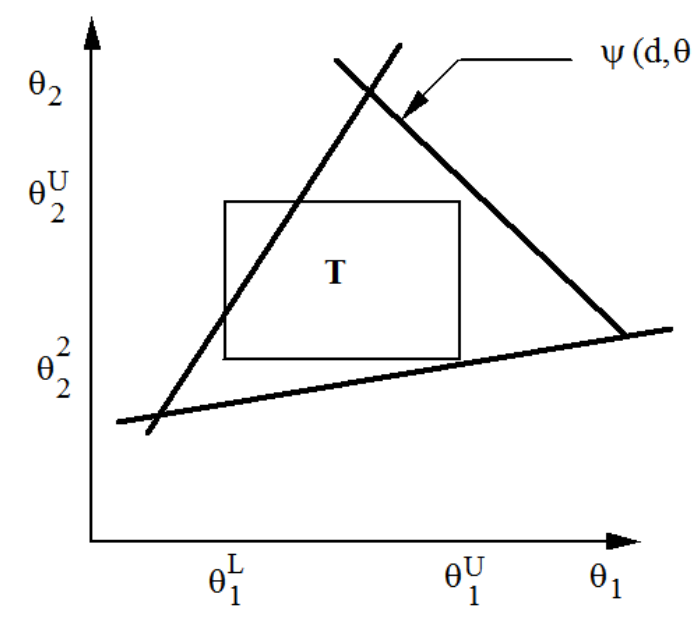

b) Infeasible design $\chi(d)>0$

Fig. 4. Regions of feasible operation for feasible and infeasible design (flexibility test problem)

In order to determine whether we can attain operation in the parameter range of interest,

$\theta \in T=\left\{\theta: \theta^{L} \leq \theta \leq \theta^{U}\right\}$

we need to establish whether $\psi(d, \theta) \leq 0$ for all $\theta \in T$. This is also equivalent to stating whether the maximum value of $\psi(d, \theta)$ is less or equal than zero in the range of $\theta$. Hence, the flexibility test problem can be formulated as:

$\chi(d)=\max _{\theta \in T} \psi(d, \theta)$

where $\chi(d)$ corresponds to the flexibility function of design $d$ over the range $T$. If $\chi(d) \leq 0$, it then means that feasible operation can be attained over the parameter range $T$ (see Fig. 4a). If $\chi(d)>0$ it means that at least for part of the range of $T$, feasible operation cannot be achieved (see Fig. 4b). Also the value of $\theta$ that is determined in (10) can be regarded as critical for the parameter range $T$ since it is the one where the feasibility of operation is the smallest $(\chi(d) \leq 0)$ or where maximum constraint violation occurs $(\chi(d)>0)$.

Finally, by substituting (7) in (10), the general mathematical formulation of the flexibility test problem yields,

$\chi(d)=\max _{\theta \in T} \min _{z} \max _{j \in J} f_{j}(d, z, \theta)$

\subsection{Flexibility index problem}

The drawback in the flexibility test problem is that it only determines whether a design does or does not have the flexibility to operate over the specified parameter range $T$. It is desirable to develop a quantitative measure that will indicate how much flexibility can actually be achieved in the given design (Swaney \& Grossmann, 1985). To address this question, we define a variable parameter range:

$T(\delta)=\left\{\theta: \theta^{N}-\delta \Delta \theta^{-} \leq \theta \leq \theta^{N}+\delta \Delta \theta^{+}\right\}$ 
where $\delta$ is a non-negative scalar variable. Note that for $\delta=1, T(1)=T$; i.e. in this case $T(\delta)$ becomes identical to the specified parameter range $T$. For $\delta<1$, it is clear that $T(\delta) \subset T$, while for $\delta>1, T(\delta) \supset T$.

We can then define the Flexibility Index, $F$, as the largest value of $\delta$ such that the inequalities $f_{j}(d, z, \theta) \leq 0, j \in J$, hold over the parameter range $T(F)$ (i.e. $\chi(d) \leq 0$ for $T(F)$ ). Mathematically, this problem can be formulated as:

$$
\begin{array}{cl}
F=\max & \delta \\
\text { s.t. } & \chi(d)=\max _{\theta \in T} \min _{z} \max _{j \in J} f_{j}(d, z, \theta) \leq 0 \\
& T(\delta)=\left\{\theta: \theta^{N}-\delta \Delta \theta^{-} \leq \theta \leq \theta^{N}+\delta \Delta \theta^{+}\right\} \\
& \delta \geq 0
\end{array}
$$

The geometric interpretation of this problem is shown in Fig. 5 where it can be seen that the rectangle $T(F)$ is the largest rectangle that can be inscribed within the region of operation. This rectangle is centered at the nominal point and its sides are proportional to the expected deviations, $\Delta \theta^{+}, \Delta \theta^{-}$. The flexibility index also indicates the actual parameter range that can be handled by the design; this will be given by (see Fig. 5),

$T(\delta)=\left\{\theta: \theta^{N}-F \Delta \theta^{-} \leq \theta \leq \theta^{N}+F \Delta \theta^{+}\right\}$

A value $F=1$ implies that the design has exactly the flexibility to satisfy the constraints over the set $T$. A value $F>1$ implies that the design exceeds the flexibility requirements; a value $F<1$ supplies the fractional deviation that can actually be handled for any of the expected deviations. Finally, the value of $\theta$ determined by (13) corresponds to the critical parameter point, $\theta^{C}$, that limits flexibility (see Fig. 5). 


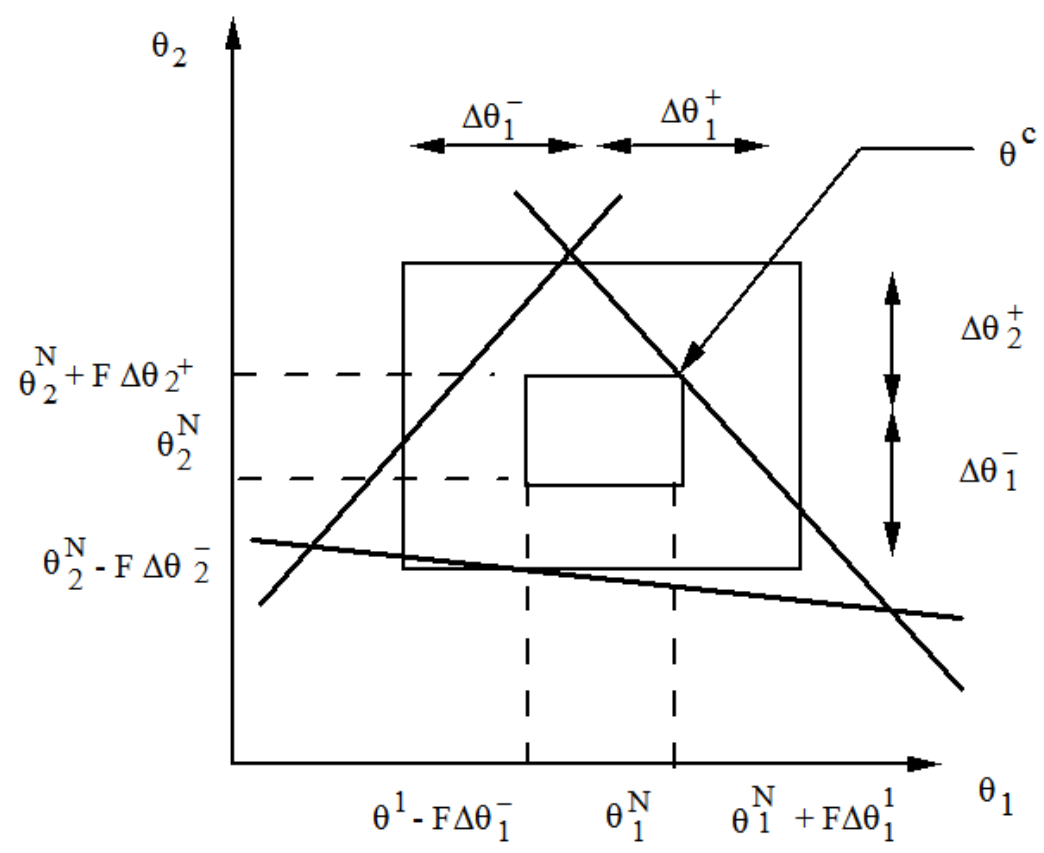

Fig. 5. Geometrical representation of parameter range $T(F)$ with flexibility index $F$.

\subsection{Vertex solution methods}

The solution of (11) for the flexibility test problem and of (13) for the flexibility index problem can be greatly simplified for the case when the critical points correspond to vertices or extreme values of the parameter sets $T$ and $T(F)$, respectively (Halemane \& Grossmann, 1983).

Consider first the flexibility test problem, and let $\theta^{k}, k \in V$, represent the vertices of the set $T$. Then, (10) reduces to:

$\chi(d)=\max _{k \in V} \psi\left(d, \theta^{k}\right)$

Note that $\psi\left(d, \theta^{k}\right)$ can be evaluated through the optimization problem in (8) at the vertex $\theta^{k}$. Hence, the following algorithm can be applied:

Step 1. For each vertex $\theta^{k}, k \in V$, solve the optimization problem:

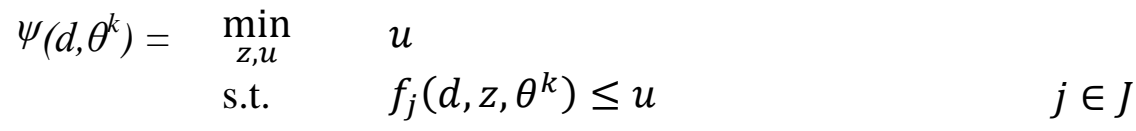

Step 2. Set $\chi(d)=\max _{k \in V}\left\{\psi\left(d, \theta^{k}\right)\right\}$

If $\chi(d) \leq 0$ then design is feasible to operate over the set $T$; otherwise if $\chi(d)>0$ it is not.

A similar procedure can be applied for the flexibility index problem. First, note that in (13) $\chi(d)=0$ at the optimal solution since the critical point in this case will always lie on the 
boundary (see Fig. 5). Let $\Delta \theta^{k}, k \in V$, denote the vertex directions from the nominal point to the vertex points in $T$. Then, the maximum deviation $\delta^{k}$ to the boundary along $\Delta \theta^{k}$ will be given by the optimization problem:

$$
\begin{aligned}
\delta^{k}=\max _{z, \delta} & \delta \\
\text { s.t. } & f_{j}\left(d, z, \theta^{k}\right) \leq 0 \quad j \in J \\
& \theta^{k}=\theta^{N}+\delta \Delta \theta^{k}
\end{aligned}
$$

From among the parameter rectangles $T\left(\delta^{k}\right), k \in V$, it is clear that only the smallest one can be totally inscribed within the feasible region (Kabatek \& Swaney, 1992). Hence,

$F=\min _{k \in V}\left\{\delta^{k}\right\}$

Thus, the following algorithm applies,

Step 1. Solve the optimization problem in (17) for each vertex $k \in V$.

Step 2. Set $F=\min _{k \in V}\left\{\delta^{k}\right\}$

It can be shown that only under some convexity conditions (see Swaney \& Grossmann, 1985) for the constraint functions $f_{j}, j \in J$, the critical points will always correspond to vertices (e.g. linear functions). For most cases however, when these conditions are not met we will often still have vertex critical points. A second reason is that even if critical points are vertices, we may be faced with the problem of having to analyze an exponential number of vertices $\left(2^{p}\right)$. We present next a method that can overcome these problems.

\subsection{Active-set method}

The flexibility test in problem (10) and the flexibility index in problem (13) can be formulated as mixed-integer optimization problems (Grossmann \& Floudas, 1987). Let us consider first problem (10), the flexibility test, which with (7) becomes,

$$
\begin{aligned}
\chi(d)=\max _{\theta \in T} & \psi(d, \theta) \\
\text { s.t. } & \psi(d, \theta)=\min _{z} \max _{j \in J} f_{j}(d, z, \theta)
\end{aligned}
$$

The above is a two-level optimization problem since it involves as a constraint the min-max problem for the function $\psi(\cdot)$. In order to convert this constraint into algebraic equations, let us consider the Karush-Kuhn-Tucker conditions of the function $\psi(d, \theta)$ as defined by the problem in (8). These conditions yield:

$\sum_{j \in J} \lambda_{j}=1$ 
$\sum_{j \in J} \lambda_{j} \frac{\partial f_{j}(d, z, \theta)}{\partial z}=0$

$\lambda_{j}\left[f_{j}(d, z, \theta)-u\right]=0$

$j \in J$

$\lambda_{j} \geq 0 ; \quad f_{j}(d, z, \theta)-u \leq 0$

$j \in J$

where $\lambda_{j}$ are the Lagrange multipliers for the constraints $f_{j}(d, z, \theta)-u \leq 0$ in (8). Since at the optimal solution of (8), $\psi(d, \theta)=u$, we can reformulate (19) as a single optimization problem.

$$
\begin{array}{rl}
\chi(d)=\max _{\theta \in T} & u \\
\text { s.t. } & \text { Constraints in (20) }
\end{array}
$$

The complementary conditions in (20c) imply making discrete choices of those constraints that become active in (8), i.e. $f_{j}(d, z, \theta)=u$. That is, if $\lambda_{j}=0, f_{j}-u<0$, constraint $j$ is inactive. These discrete choices can be modeled as follows.

Let $s_{j} \geq 0$, be the slack of constraint $f_{j}(d, z, \theta)-u \leq 0$, such that

$f_{j}(d, z, \theta)+s_{j}=u \quad j \in J$

Also let $y_{j}$ be a 0-1 variable defined as follows:

$y_{j}= \begin{cases}1 & \text { if constraint } f_{j}-u=0 \\ 0 & \text { otherwise }\end{cases}$

This binary variable can be related to $s_{j}$ and $\lambda_{j}$ by the logical inequalities:

$s_{j} \leq U\left(1-y_{j}\right)$

$\lambda_{j} \leq y_{j}$

$j \in J$

where $U$ is a valid upper bound for the slacks. Note that if $y_{j}=1$, it implies $s_{j}=0, \lambda_{j} \leq 1$; if $y_{j}=0$, it implies $0 \leq s_{j} \leq U, \lambda_{j}=0$. In other words the inequalities in (23) are equivalent to the conditions in (20c).

Furthermore, it can be shown that if the gradients $\frac{\partial f_{j}}{\partial z}, j \in J$, are linearly independent (Swaney \& Grossmann, 1985), then there will be $n_{z}+1$ active constraints in (21), where $n_{z}$ is the dimensionality of the control variables $z$. Hence, we can set

$y_{j}=n_{z}+1 \quad j \in J$

By then considering equations (22), (24), (25) in place of (20c) and (20d), problem (21) can be posed as the following mixed-integer optimization problem: 


$$
\begin{array}{clc}
\max _{u, \theta, z, \lambda_{j}, s_{j}, y_{j}} & u & \\
\text { s.t. } & f_{j}(d, z, \theta)+s_{j}=u & \\
& \sum_{j \in J} \lambda_{j}=1 & \\
& \sum_{j \in J} \lambda_{j} \frac{\partial f_{j}(d, z, \theta)}{\partial z}=0 & j \in J \\
& s_{j} \leq U\left(1-y_{j}\right) & j \in J \\
& \lambda_{j} \leq y_{j} & \\
& y_{j}=n_{z}+1 & \\
& \theta^{L} \leq \theta \leq \theta^{U} & j \in J \\
& \lambda_{j}, s_{j} \geq 0 ; \quad y_{j} \in\{0,1\} & j \in J
\end{array}
$$

Note that in the above formulation all the variables, $u, \theta, z, \lambda_{j}, s_{j}, y_{j}, j \in J$ appear as variables for the optimization since these are constrained to solve the problem for $\psi(d, \theta)$ through the constraints. There are several interesting features about the formulation in (26):

a) If $f_{j}$ is linear in $z$ and $\theta$ (26) corresponds to an MILP problem (note $\frac{\partial f_{j}}{\partial z}$ is constant for this case). Otherwise it corresponds to an MINLP.

b) No enumeration of vertices is required, and therefore many uncertain parameters can be handled.

c) The derivation of problem (26) does not require the assumption of vertex critical points. Hence, non-vertex critical points can be predicted such as in the motivating heat exchanger network problem (Grossmann and Floudas, 1987).

A similar formulation can be derived for the flexibility index problem by reformulating (13) as the minimum $\delta$ to the boundary $\psi(d, \theta)$. That is,

$$
\begin{aligned}
F= & \min \delta \\
& \text { s.t. } \quad \psi(d, \theta)=0
\end{aligned}
$$

Since the constraint $\psi(d, \theta)=0$ implies setting $u=0$ in problem (26) and from the deviation of the variable parameter range in (12), the flexibility index problem can be posed as the following mixed-integer optimization problem:

$$
\begin{array}{cl}
F=\min _{\delta, \lambda_{j}, s_{j}, y_{j}} & \delta \\
\text { s.t. } & f_{j}(d, z, \theta)+s_{j}=0 \quad j \in J
\end{array}
$$




$$
\begin{array}{ll}
\sum_{j \in J} \lambda_{j}=1 & \\
\sum_{j \in J} \lambda_{j} \frac{\partial f_{j}(d, z, \theta)}{\partial z}=0 & \\
s_{j} \leq U\left(1-y_{j}\right) & j \in J \\
\lambda_{j} \leq y_{j} & j \in J \\
y^{j}=n_{z}+1 & \\
\theta^{N}-\delta \Delta \theta^{-} \leq \theta \leq \theta^{N}+\delta \Delta \theta^{+} & \\
\delta, \lambda_{j}, s_{j} \geq 0 ; \quad y_{j} \in\{0,1\} & j \in J
\end{array}
$$

This problem has again similar features as the flexibility test problem in (26).

\subsection{Special cases and extension for flexibility analysis}

The previous sections assumed that the uncertain parameters are independent. The case of correlated parameters can be easily handled by specifying these in algebraic equations $r(\theta)=0$, which are added as constraints in formulations (26) or (28).

For the case when there are no control variables (i.e. $n_{Z}=0$ ) the formulations are simplified. Consider for instance problem (26) for the flexibility test. If $n_{Z}=0$, the stationary conditions in (20) are not required. Hence problem (26) reduces to:

$$
\begin{array}{clc}
\chi(d)=\max _{u, \theta, s_{j}, y_{j}} & u & \\
\text { s.t. } & f_{j}(d, \theta)+s_{j}=u & j \in J \\
& s_{j} \leq U\left(1-y_{j}\right) & j \in J \\
& y_{j}=1 & j \in J \\
& \theta^{L} \leq \theta \leq \theta^{U} & \\
& s_{j} \geq 0 ; \quad y_{j} \in\{0,1\} & j \in J
\end{array}
$$

Since in the above formulation only one constraint can be active, we can easily decompose the solution to this problem by setting $s_{j}=0$ and maximizing $u=f_{j}(d, \theta)$ for each constraint $j$. That is the problem reduces to:

Step 1. For each constraint $j \in J$, solve: $\quad u_{j}=\max _{\theta^{L} \leq \theta \leq \theta^{U}} f_{j}(d, \theta)$

Step 2. Set $\chi(d)=\max _{j \in J}\left\{u_{j}\right\}$ 
In a similar fashion, it can easily be shown that for $n_{Z}=0$ the problem for the flexibility index reduces from (28) to:

Step 1. For each constraint $j \in J$, solve: $\delta_{j}=\max _{\delta, \theta} \quad \delta$

$$
\begin{array}{ll}
\text { s.t. } & f_{j}(d, \theta)=0 \\
& \theta^{N}-\delta \Delta \theta^{-} \leq \theta \leq \theta^{N}+\delta \Delta \theta^{+}
\end{array}
$$

Step 2. Set $F=\min _{j \in J}\left\{\delta^{j}\right\}$

That is, for each constraint we determine the closest displacement $\delta_{j}$ to the boundary $f_{j}(d, \theta)=0$, and then set the index $F$ to the smallest of all the displacements.

Finally, we consider the case where we would like to explicitly keep the performance equations to avoid the algebraic elimination of the state variables. In the case when there are no control variables this is straightforward as we then simply have to include the equations $h_{i}(d, x, \theta)=0$, $i \in I$, in the optimization problems. For example, for the flexibility test, in the two-step procedure $u_{j}$ can be determined as:

$$
\begin{aligned}
u^{j}=\max _{\theta, x} & g_{j}(d, x, \theta) \\
\text { s.t. } & h_{i}(d, x, \theta)=0 \\
& \theta^{L} \leq \theta \leq \theta^{U}
\end{aligned} \quad i \in I
$$

The feasibility function $\psi(d, \theta)$ in (8) must be redefined as:

$$
\begin{array}{lll}
\psi(d, \theta)=\max _{u, z, x} & u & \\
\text { s.t. } & h_{i}(d, x, \theta)=0 & i \in I \\
& g_{j}(d, x, \theta) \leq u & j \in J
\end{array}
$$

This formulation would then be used for the vertex search method for the flexibility test. For the mixed-integer formulation in (26), the Karush-Kuhn-Tucker conditions of problem (31) must be included. Using a similar reasoning as in (26) the flexibility test problem corresponds to:

$$
\begin{array}{lll}
\chi(d)=\max _{u, \theta, z, \lambda_{j}, \mu_{i}, s_{j}, y_{j}} & u \\
\text { s.t. } & h_{i}(d, x, z, \theta)=0 & i \in I \\
& g_{j}(d, x, z, \theta)+s_{j}-u=0 & \\
& \sum_{j \in J} \lambda_{j}=1 & \\
& \sum_{i \in I} \mu_{i} \frac{\partial h_{i}(d, x, z, \theta)}{\partial z}+\sum_{j \in J} \lambda_{j} \frac{\partial g_{j}(d, x, z, \theta)}{\partial z}=0
\end{array}
$$




$$
\begin{array}{lr}
\sum_{i \in I} \mu_{i} \frac{\partial h_{i}(d, x, z, \theta)}{\partial x}+\sum_{j \in J} \lambda_{j} \frac{\partial g_{j}(d, x, z, \theta)}{\partial x}=0 & \\
s_{j} \leq U\left(1-y_{j}\right) & j \in J \\
\lambda_{j} \leq y_{j} & j \in J \\
y^{j}=n_{z}+1 & \\
\theta^{L} \leq \theta \leq \theta^{U} & \\
\lambda_{j}, s_{j} \geq 0 ; \quad y_{j} \in\{0,1\} & j \in J
\end{array}
$$

where $\mu_{i}$ are Lagrange multipliers to the equality constraints in (37) that are unrestricted in sign. Note that in (32) we have the advantage of not having to eliminate equations although we face a problem larger in size than in (26). Similar extensions can be performed for the flexibility index problem in (28).

\subsection{Optimal design of flexible processes}

As for design optimization problems they involve the selection of the design variables $d$ so as to minimize cost and either a) satisfy the flexibility test (11), or b) maximize the flexibility measure as given by (13), where the latter problem gives rise to a multi-objective optimization problem. Most of the initial work in design under uncertainty (Johns et al., 1976; Malik \& Hughes, 1979) considered a joint probability distribution function $j(\theta)$ for the continuous uncertain parameters $\theta$ in order to determine the design optimization by minimizing the expected value of the cost using a two-stage strategy:

$$
\min _{d} \underset{\theta \in T(F)}{E}\left[\min _{z} c(d, z, \theta) \mid f(d, z, \theta) \leq 0\right]
$$

The reason the above is denoted as a two-stage strategy in analogy to stochastic programming (Birge \& Louveaux, 2011) is because the design variables are chosen in stage 1 and remain fixed during stage 2 during which the control variables $z$ are adjusted depending on the realizations of the parameters $\theta$. In order to handle infeasibilities in the inner minimization, one approach is to assign penalties for the violation of constraints (e.g. $C(d, z, \theta)=\bar{C}(d, z, \theta)$ if $f(d, z, \theta)>0$ ). This however can lead to discontinuities. The other approach is to enforce feasibility for a specified flexibility index $F$ (e.g. see Halemane \& Grossmann, 1993) through the parameter set:

$T(F)=\left\{\theta: \theta^{N}-F \Delta \theta^{-} \leq \theta \leq \theta^{N}+F \Delta \theta^{+}, r(\theta) \leq 0\right\}$

In this case (27) is formulated as:

$$
\begin{array}{cl}
\min _{d} \underset{\theta \in T(F)}{E} & {\left[\min _{z} C(d, z, \theta) \mid f(d, z, \theta) \leq 0\right]} \\
\text { s.t. } & \max _{\theta \in T(F)} \psi(d, \theta) \leq 0
\end{array}
$$


A particular case of (34) is when only a discrete set of points $\theta^{k}, k=1, \ldots, K$ are specified which then gives rise to the optimal design problem,

$$
\begin{aligned}
\min _{d} & \sum_{k=1}^{K} w^{k} C\left(d, z^{k}, \theta^{k}\right) \\
\text { s.t. } & f\left(d, z^{k}, \theta^{k}\right) \leq 0 \\
\text { where } & \sum_{k=1}^{K} w^{k}=1
\end{aligned}
$$

and $w^{k}$ are weights that are assigned to each point $\theta^{k}$. Problem (35) can be interpreted as a multiperiod design problem, which is an important problem in its own right for the design of flexible chemical plants (see Grossmann \& Sargent, 1979; Varvarezos et al. 1992, 1994). As shown by Grossmann \& Sargent (1978) problem (35) can also be used to approximate the solution of (34). This is accomplished by selecting an initial set of points $\theta^{k}$, solving problem (35), and verifying its feasibility over $T(F)$ by solving problem (11) or (13). If the design is feasible the procedure terminates. Otherwise the critical point obtained from the flexibility evaluation is included to the set of $K \theta$ points and the solution of (35) is repeated. Commonly only one or two major iterations must be performed to achieve feasibility with this method. Other approaches for the design problem can be found in Pistikopoulos \& Grossmann $(1988,1989)$.

It is also interesting to point out that for the case when there are no control variables $z$, and the cost function $C(d)$ depends only on $d$, problem (34) can be interpreted as a robust optimization problem in which the goal is to find the design variables $d$ so as to meet the inequalities $f(d, \theta) \leq 0, \forall \theta \in T$ (Ben-Tal et al., 2009). In that sense, problem (34) can be regarded as a more general formulation than robust optimization since it involves the recourse or control variables $z$, as well as the joint distribution function $j(\theta)$ over the uncertainty set $T(F)$.

\section{Fundamental concepts for dynamic resiliency}

As was illustrated in the motivating examples section, dynamic resiliency was introduced with some counterintuitive observations. For instance, longer dead times can sometimes improve plant dynamic resiliency. A basic question was: why can perfect controllers not be implemented? The key idea was to recognize some inherent characteristics of a system, namely, time delays, non-minimum phase elements, constraints on manipulated variables, and model uncertainty. The key contribution by Manfred Morari and his group was to assess quantitatively the effect of each of them. This was accomplished with a brilliant series of 9 papers on Design of Resilient Processing Plants. Each of the 9 parts is listed in Table 1.

Table 1. Series of nine papers by Morari and coworkers on design of resilient processing plants. 
1. Process Design Under Consideration of Dynamic Aspects

Lenhoff AM; Morari M

Chemical Engineering Science Volume: 37 Issue: 2 Pages: 245-258 (1982)

2. Design And Control of Energy Management-systems

Marselle DF; Morari M; Rudd Df

Chemical Engineering Science Volume: 37 Issue: 2 Pages: 259-270 (1982)

3. A General Framework for The Assessment Of Dynamic Resilience Morari M

Chemical Engineering Science Volume: 38 Issue: 11 Pages: 1881-1891 (1983)

4. Some New Results on Heat-exchanger Network Synthesis

Saboo AK; Morari M

Chemical Engineering Science Volume: 39 Issue: 3 Pages: $579-592$ (1984)

5. The Effect of Deadtime on Dynamic Resilience

Holt BR; Morari M

Chemical Engineering Science Volume: 40 Issue: 7 Pages: 1229-1237 (1985)

6. The Effect Of Right-half-plane Zeros on Dynamic Resilience

Holt BR; Morari M

Chemical Engineering Science Volume: 40 Issue: 1 Pages: 59-74 (1985)

7. Design of Energy Management-system for Unstable Reactors - New Insights Morari M; Grimm W; Oglesby MJ; et al.

Chemical Engineering Science Volume: 40 Issue: 2 Pages: 187-198 (1985)

8. A Resilience Index for Heat-exchanger Networks

Saboo AK; Morari M; Woodcock DC

Chemical Engineering Science Volume: 40 Issue: 8 Pages: 1553-1565 (1985)

9. Effect of Model Uncertainty on Dynamic Resilience

Skogestad S; Morari M

Chemical Engineering Science Volume: 42 Issue: 7 Pages: 1765-1780 (1987)

For ease of exposition, we present the mathematical treatment of dynamic resiliency for SingleInput-Single-Output (SISO) systems and note that more details and extensions to the multivariable case are available in Morari (1983). We assume that the system can be represented by a transfer matrix $G(s)$, i.e.:

$y(s)=G(s) u(s)$

where $y(s) \in R^{n}$ are the outputs to be controlled and $u(s) \in R^{m}$ are manipulated variables. Fig. 6 shows the feedback control structure analyzed in this section, where $C(s) \in R^{m \times n}$ is the controller transfer matrix, $y_{s}(s) \in R^{n}$ the set points and $d \in R^{n}$ the disturbances.

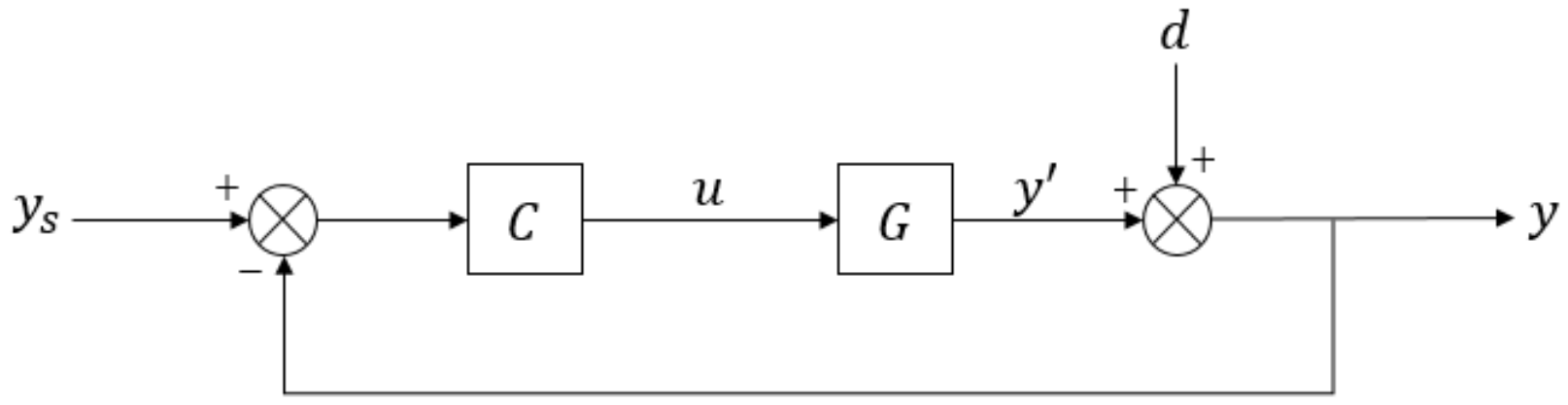

Fig. 6. Typical feedback control structure.

In order to achieve perfect regulatory control, i.e. $y(t)=y_{s}(t)$, at all times and for all disturbances affecting the system, that is $y^{\prime}(s)=y_{s}(s)-d(s)$, we require 
$u(s)=G^{-1}(s)\left(y_{s}(s)-d(s)\right)$

where $G^{-1}(s)$ is the right inverse of $G(s)$. A perfect controller is only possible if $G(s)$ has a right inverse. From the block diagram in Fig. 6, we find that

$u(s)=C(s)(I+G(s) C(s))^{-1}\left(y_{s}(s)-d(s)\right)$

where $C(s)(I+G(s) C(s))^{-1}$ is called the Closed-Loop Controller Transfer Matrix (CLCTM). Therefore, the objective is to make the CLCTM equal to $G^{-1}(s)$ without imposing constraints on the structure of $C(s)$.

Instead of proceeding with the analysis using such an indirect expression of $G^{-1}(s)$, it is more convenient to introduce the Internal Model Control (IMC) structure whose block diagram is shown in Fig. 7, where $\bar{G}(s)$ is an approximate model of the system, since the system itself is never known exactly. We remark that the two representations are equivalent as expressed in the following relations:

$G_{C}=C(I+\bar{G} C)^{-1}$

$C=G_{C}\left(I-\bar{G} G_{C}\right)^{-1}$

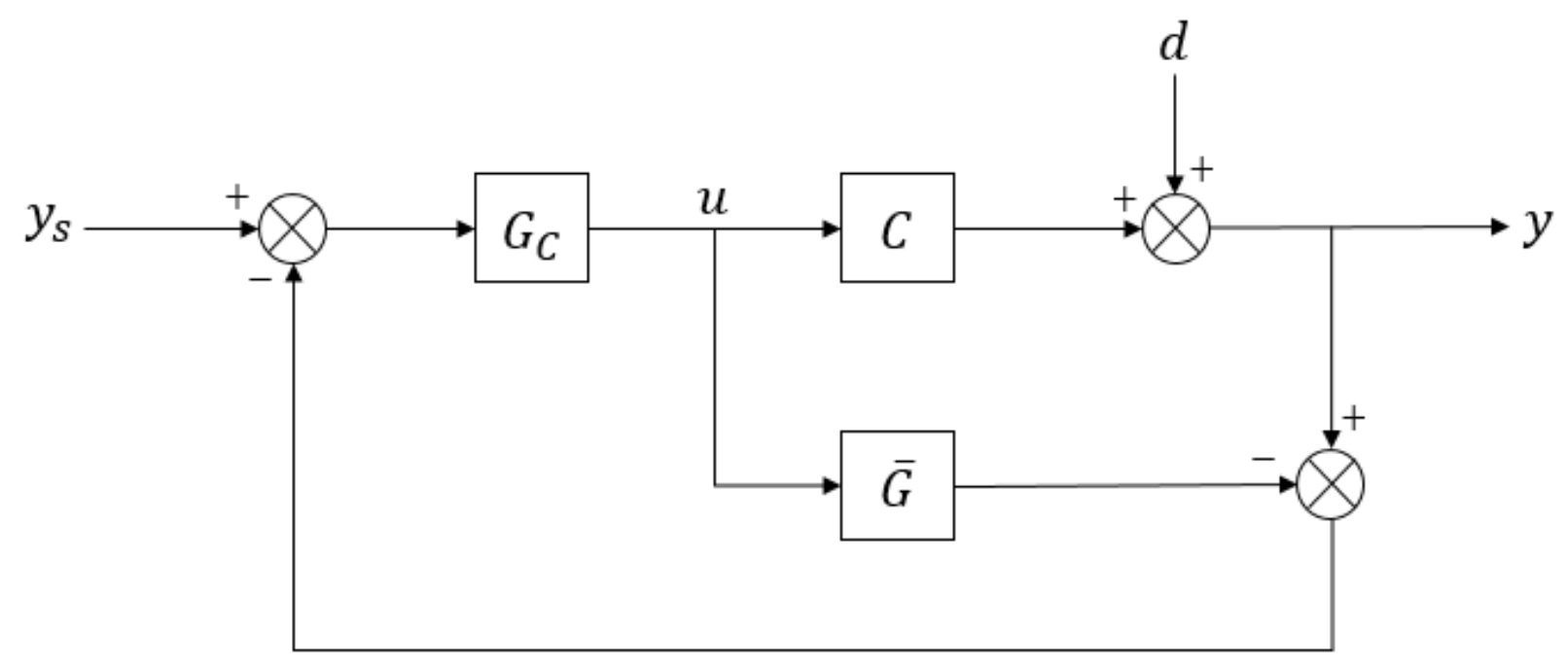

Fig. 7. Internal Model Control (IMC) structure.

Let us restate our goal. We want to select the controller $G_{C}(s)$ to be "close" to $\bar{G}^{-1}$ subject to the constraints that it be realizable (to allow physical implementation) and stable (to guarantee closed-loop stability). $\bar{G}^{-1}$ is not realizable and/or stable if $\bar{G}(s)$ is a non-minimum phase (NMP), i.e. it contains time delays and/or zeros in the right-half plane (RHP). If $\bar{G}(s)$ is an NMP, then it can be factored into an invertible part, $\bar{G}_{-}(s)$, and a non-invertible part, $\bar{G}_{+}(s)$ :

$\bar{G}(s)=\bar{G}_{+}(s) \bar{G}_{-}(s)$ 
such that $\left\|\bar{G}_{+}(s)\right\|=1$ and $\bar{G}_{-}(s)^{-1}$ is realizable and stable. Therefore, we choose $G_{C}(s)=$ $\bar{G}_{-}(s)^{-1}$.

In order to study how constraining the manipulated variables as $\|u(s)\| \leq\left\|u_{\max }\right\|$ affects resiliency, assuming a perfect model $\bar{G}(s)=G(s)$, from Fig. 6 we obtain that:

$\left\|y_{s}(s)-d\right\| \leq\|\bar{G}(i \omega)\|\left\|u_{\max }\right\|$

which, if we normalize $u(s)$ appropriately so that $\left\|u_{\max }\right\|=1$, can be interpreted as the amplitude ratio plot for the open-loop system, $\|\bar{G}(i \omega)\|$, is also a plot of the maximum disturbance, $\left\|y_{s}(s)-d\right\|$, that can be handled by the closed-loop system when a "perfect" controller is employed.

However, in practice and particularly at high frequencies, the disturbance often exceeds the bound set by $\|\bar{G}(i \omega)\|$, and when it happens the control loop performance deteriorates as the "perfect" controller cannot function properly due to the saturation of the manipulated variables. A "practical" controller will have to depart from the "perfect" controller at high frequencies. The point of departure can be used as a measure of the effect of the constraints on manipulated variables on closed-loop performance and resiliency assessment.

Thus far we have assumed a perfect model $(G=\bar{G})$. In practice, plant-model mismatch may be caused by uncertainties and nonlinearities cannot be neglected. A reasonable approach for system uncertainty is to assume that $G(s)$ is somewhere in a "ball" of radius $l(\omega)$ around model $\bar{G}(s)$, that is (see Fig. 8 for block diagram):

$G(s)=\left(1+L_{0}(s)\right) \bar{G}(s)$

where $L_{0}(s)$ is constrained as follows

$\frac{\|G(i \omega)-\bar{G}(i \omega)\|}{\|\bar{G}(i \omega)\|}=\left\|L_{0}(i \omega)\right\| \leq l(\omega)$

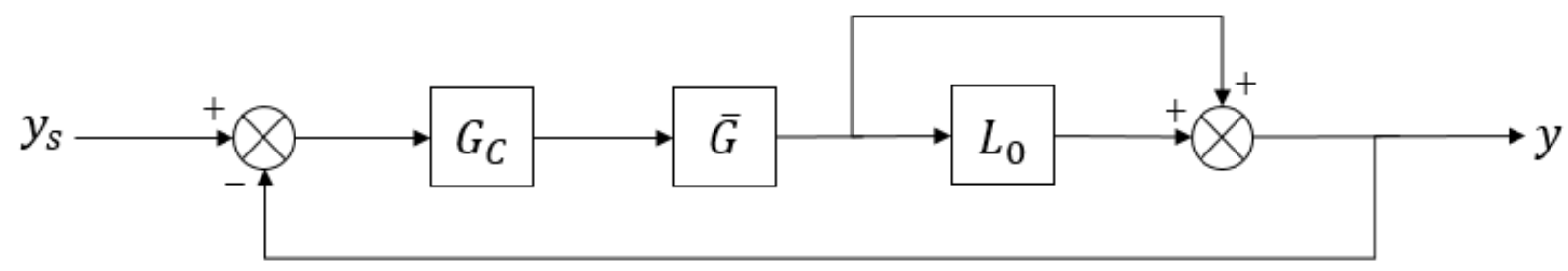

Fig. 8. Internal Model Control structure for multiplicative model uncertainties occurring at the model output

Hence, a necessary and sufficient condition for "robustness", i.e. closed-loop stability under system variations, is given by

$\left\|G_{C}(i \omega) \bar{G}(i \omega)\right\|<\frac{1}{l(\omega)}$ 
As before, we select $G_{C}(s)=\bar{G}_{-}(s)^{-1}$ and the robustness condition becomes

$l(\omega)<1$

The above condition implies that the system is only closed-loop stable if the uncertainty radius does not exceed the unity. In reality, this will not be guaranteed for high frequencies. Therefore, a dynamic compensator or a "filter", $F(s)$, is introduced to render the controller more robust. It does so by lowering the $\left\|G_{C}(s)\right\|$ at high frequencies.

$G_{C}(s)=\bar{G}_{-}(s)^{-1} F(s)$

with $F(0)=1$. Thus, aside physically constraining the control action, model uncertainty limits the frequency range over which a "perfect" control is achievable.

In summary, resiliency of single-variable systems is limited by three factors as enumerated below.

1. $\quad$ Non-minimum phase (NMP) elements, i.e. time delays and RHP zeros;

2. Physical constraints on the manipulated variables, and

3. Plant-model mismatch, which generally becomes severe at frequencies with small amplitude ratios. The amplitude ratio plot of the open-loop system provides all the information needed.

For multivariable systems, the main indicator of the sensitivity of the control structure to plantmodel mismatch is the condition number of $\bar{G}(i \omega)$ at $\omega=0$, which is denoted by $\gamma(0)$. The condition number of a matrix is the ratio between its largest and smallest singular values, $\sigma_{M}(\bar{G})$ and $\sigma_{m}(\bar{G})$, respectively; therefore, the larger the condition number the more sensitive the control system is to process variations. In the example involving the thermally-coupled distillation columns, the $\gamma(0)$ for Structure 1 is about three orders of magnitude larger than that of Structure 2, thus confirming the better control performance of Structure 2 in the simulation study.

\section{Recent extensions in flexibility analysis}

The pioneering research work by Manfred Morari on resiliency that in turn motivated the formulations by Grossmann and co-workers have seen recent extensions as described in the next sections.

\subsection{Flexible design with confidence intervals and process variability}

Rooney \& Biegler (2001) extended the mathematical foundations of Flexibility Analysis in steady-state problems to include nonlinear confidence intervals of the uncertain model parameters. In particular, the authors proposed using the likelihood ratio test to derive the confidence regions of the estimated model parameters. This method is an improvement over the deviation of individual and elliptical confidence intervals. The likelihood ratio test is defined as follows: 
$L R=2\left[L\left(\theta^{*}\right)-L(\theta)\right] \leq \eta \chi_{1-\alpha, p}^{2}$

where $L$ is the log-likelihood function, $\eta$ is the Bartlett correction factor, and $\chi_{1-\alpha, p}^{2}$ is the chisquared test statistic for a significance level of $1-\alpha$ and $p$ degrees of freedom.

As described previously in the paper, a flexible design is obtained in a two-stage optimization approach. In the first stage, the multiperiod problem in Eq. (35) is solved for the design variables, $d$. The solution of this problem is represented by $\bar{d}$ and is optimal for the discretization points chosen. The multiperiod problem is followed by the flexibility problem in Eq. (11), which is solved using the active-set approach of Grossmann \& Floudas (1987) defined in Equation (26).

The initial values of $\theta^{k}$ are obtained by solving the off-line problem in Equation (50), in which we simply find the point in the confidence region that lies furthest away (in Euclidean space) from the optimal estimates.

$$
\begin{array}{ll}
\max & \sum_{i=1}^{p}\left[\theta^{k}-\left(\theta^{k}\right)^{*}\right]^{2} \\
\text { s.t. } & 2\left[L\left(\theta^{*}\right)-L(\theta)\right] \leq \eta \chi_{1-\alpha, p}^{2}
\end{array}
$$

In a subsequent paper, Rooney \& Biegler (2004) further extended the analysis to include unmeasured process variability. The uncertain parameters, $\theta \in \Theta$, are distinguished as follows:

- Unmeasured uncertain parameters, $\theta^{u} \in \Theta^{u}$, which are never known exactly. Therefore, no control or recourse action can be applied to them. Examples include kinetic constants and unobservable disturbances.

- Measured uncertain parameters, $\theta^{m} \in \Theta^{m}$, which are not known at the design stage, but for which control action can be taken since they can be measured at the operating stage. Examples include feed flow rates, pressures and temperatures, and product demands.

Therefore, the multiperiod problem in Equation (35) is extended to include both sources of uncertain parameters.

$$
\begin{aligned}
& \min _{d, z} \quad C(d) \\
& \left.\begin{array}{cc}
\text { s.t. } & f_{i, k}\left(x_{i, k}, z_{k}, d, \theta_{i}^{m}, \theta_{k}^{u}\right) \leq 0 \\
& \theta_{i}^{m} \in \Theta^{m}, \quad \theta_{k}^{u} \in \Theta^{u}
\end{array}\right\} i \in \bar{I}, k \in \bar{K}
\end{aligned}
$$

where $i$ and $k$ are the discretization points of $\theta^{m}$ and $\theta^{u}$, respectively, and $\bar{I}$ and $\bar{K}$ are index sets that represent the critical points that are found if the constraints cannot be satisfied in the second stage.

The flexibility test is extended to account for unmeasured process variability, $\theta^{u}$. As before, for a given solution $\bar{d}$ obtained by solving the first-stage problem in Eq. (50), the flexibility problem is defined as the nested optimization problem as follows: 
$\chi(\bar{d})=\max _{\theta^{m} \in \Theta^{m}} \min _{z} \max _{\theta^{u} \in \Theta^{u}} \max _{j} f_{j}\left(x, z, \bar{d}, \theta^{m}, \theta^{u}\right)$

The multilevel and nondifferentiable optimization problem in Eq. (51) is cast as a single-level smooth optimization problem through the use of a modified KS function (Kreisselmeier \& Steinhauser, 1979) defined below and application of KKT conditions. More details on the reformulation can be found in the original paper.

$\widehat{K S}\left(x, z, \bar{d}, \theta^{m}, \theta^{u}, \rho\right)=\frac{1}{\rho} \ln \left\{\frac{\sum_{j}^{|J|} \exp \left[\rho g f_{j}\left(x, z, \bar{d}, \theta^{m}, \theta^{u}\right)\right]}{|J|}\right\}$

\subsection{Simplicial approximation to feasibility limits}

In contrast to finding the hyperrectangle corresponding to the flexibility index (Fig. 5), Goyal \& Ierapetritou (2002) presented an approach based on the ideas of inner and outer approximation of the feasible region in order to measure the entire feasible region. However, they restricted themselves to the case of no control variables.

The inner approximation of the feasible region, $R$, is represented by a polyhedron made up of $n$ dimensional simplices, where it is assumed that the constraint functions are locally convex. It provides a lower bound to $R$. The algorithm is summarized in the following steps where more details can be found in the original paper. Fig. 9 shows a schematic of the algorithm.

1. Determine any $m$ points on the boundary of the feasible region, $\partial R$, where $m \geq n+1$;

2. $\quad$ Using the set of $m$ points from the previous step, construct the convex hull;

3. Given the first approximation of $\partial R$, inscribe the largest hypersphere (or hyperellipsoid by scaling the boundary points) by solving a linear programming problem;

4. Determine the largest of the faces of the polyhedron that are tangent to the inscribed hypersphere (or hyperellipsoid) by solving a linear programming problem;

5. Determine new boundary point by making a one-dimensional search in the outwards normal direction, starting at the center of the largest inscribed hypersphere (or hyperellipsoid) obtained in the previous step;

6. Add the new point to the set of boundary points and a new convex hull is formed, thus improving the approximation to $\partial R$. 


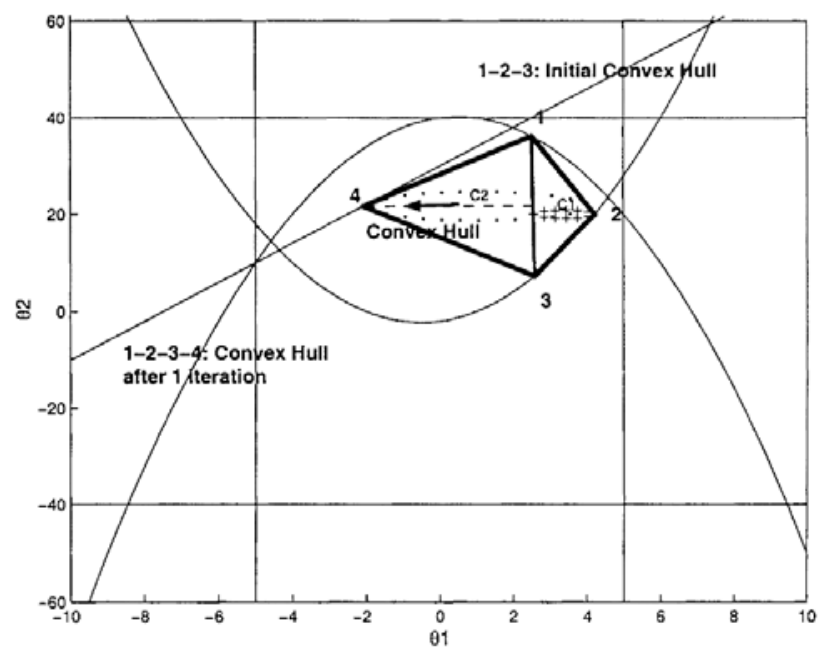

Fig. 9. Steps in simplicial approximation. Initial convex hull $(1,2,3)$ and the resulting convex hull $(1,2,3,4)$ after one iteration (Goyal \& Ierapetritou, 2002).

The outer approximation, which is a convex envelope, provides an upper bound to the feasible region, $R$. The proposed algorithm is based on the supporting hyperplane theorem of convex sets that states that if $S$ is a convex set and $p$ is a boundary point of $S$, then there exists a hyperplane containing $p$ and containing $S$ in one of its closed half-spaces. The algorithm is summarized in the following steps.

1. Use points generated by the simplicial approximation as initial boundary points;

2. Generate hyperplanes at the points from the previous step, which requires no extra optimization problems to be solved if the constraints are explicitly defined, but additional optimization problems in the form of Equation (8) must be solved in case constraints are implicit;

3. $\quad$ Obtain the points of intersection at the tangent half-planes;

4. Generate convex hull at the intersection points obtained in the previous step, thus forming the outer polytope.

Subsequent papers that build on the ideas of the simplicial approximation are by Banerjee \& Ierapetritou (2005), in which the authors consider the feasible region as an object and apply surface reconstruction ideas to capture and define the shape of the object by sampling points and constructing a polygonal representation of the feasible region, and by Boukouvala \& Ierapetritou (2012), who addressed the feasibility evaluation of black-box processes using Kriging interpolation and development of an adaptive sampling strategy in order to minimize sampling cost, while maintaining feasibility space accuracy.

\subsection{Stochastic flexibility}

Pistikopoulos \& Mazzuchi (1990) and Straub \& Grossmann (1990) addressed the problem where instead of specifying a fixed parameter set $T$, one assumes that the uncertain parameters $\theta$ are described by a joint probability distribution function $j(\theta)$. This involves the evaluation of the multiple integral 
$S F(d)=\int_{\theta: \psi(d, \theta) \leq 0} j(\theta) d \theta$

where $S F(d)$ is the stochastic flexibility for a given design, which can be interpreted as the probability that a design exhibit feasible operation while accounting for control variables that can be adjusted. Note that this integral must be evaluated over the feasible region projected in $\theta$ space (see Fig. 10). In Fig. 10 the circles represent the contours of the joint distribution function $j(\cdot)$.

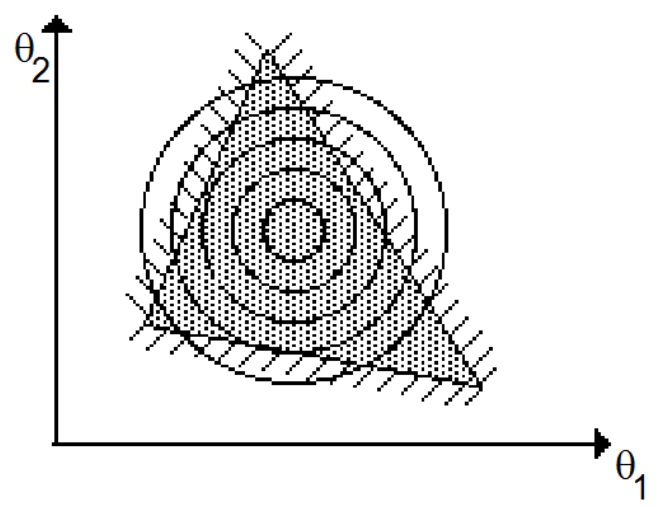

Fig. 10. SF is evaluated by integration over the shaded area.

Straub \& Grossmann (1990) have proposed a numerical approximation scheme for arbitrary distribution functions using Gaussian quadrature within the feasible region of the projected region $R(d, \theta)$ (see Fig. 11).

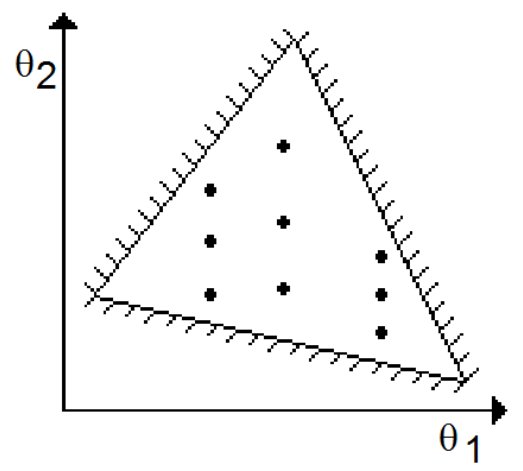

Fig. 11. Location of Quadrature Points.

The location of the quadrature periods is performed by first projecting the functions $\psi^{k}(d, \theta)$, $k=1, \ldots, N A S$, into successively lower dimensional spaces in $\theta$; i.e. $\left[\theta_{1}, \theta_{2}, \ldots, \theta_{M}\right],\left[\theta_{1}, \theta_{2}, \ldots, \theta_{M-1}\right], \ldots,\left[\theta_{1}\right]$. This is accomplished by analytically solving the problems $r=1,2, \ldots, M-1$ :

$$
\begin{aligned}
& \psi^{r+1, k}\left(d, \theta_{1}, \theta_{2}, \ldots, \theta_{M-r}\right)=\quad \min u \\
& \text { s.t. } \quad \psi^{r, k}\left(d, \theta_{1}, \theta_{2}, \ldots\right) \leq u \quad k=1, \ldots, N A S
\end{aligned}
$$


where $\psi^{1, k}=\psi^{k}(d, \theta)=f_{j}(z, d, \theta)$ for $j \in J_{A}^{k}$ and $N A S(r)$ is the number of active sets at the $r$ th state of the projection.

In the next step, lower and upper bounds are generated together with the quadrature points for each $\theta_{i}$ component in the order $\theta_{1}, \theta_{2}, \ldots, \theta_{M}$. This is accomplished by using the analytical expressions $\psi^{r, k}\left(d, \theta_{1}, \theta_{2}, \ldots, \theta_{M+r-1}\right)$ in the order $r=M, M-1, \ldots$, to determine the bounds. For instance, the bounds $\theta_{1}^{L}$ and $\theta_{1}^{U}$ are determined from the linear inequalities $\psi^{M, k}\left(d, \theta_{1}\right) \leq 0$, $k=1, \ldots, N A S(M)$. The quadrature points $\theta_{1}^{q_{1}}$ then are given by:

$\theta_{1}^{q_{1}}=\frac{v_{q_{1}}\left(\theta_{1}^{U}-\theta_{1}^{L}\right)+\theta_{1}^{U}+\theta_{1}^{L}}{2} \quad q_{1}=1, \ldots, Q P_{1}$

where $v_{q_{1}}, q_{1}=1, \ldots, Q P_{1}$ represent the location of $Q P_{1}$ quadrature points in $[-1,1]$. In the next step, bounds for $\theta_{2}$ are computed for each $\theta_{1}^{q_{1}}$ from $\psi^{M-1, k}\left(d, \theta_{1}, \theta_{2}\right) \leq 0, k=1, \ldots, N A S(M-$ $1)$. These bounds are denoted as $\theta_{2}^{L}\left(\theta_{1}^{q_{1}}\right)$ since they depend on the value of $\theta_{1}^{q_{1}}$. Quadrature points are then computed as in (54) and the procedure continues until the bounds $\theta_{M}^{L}\left(\theta_{1}^{q_{1}}, \theta_{2}^{q_{1} q_{2}}, \ldots, \theta_{M-1}^{q_{1} q_{2} \cdots q_{M-1}}\right), \theta_{M}^{U}\left(\theta_{1}^{q_{1}}, \theta_{2}^{q_{1} q_{2}}, \ldots, \theta_{M-1}^{q_{1} q_{2} \cdots q_{M-1}}\right)$ and quadrature points $\theta_{M}^{q_{1} q_{2} \cdots q_{M}}$ are determined.

The numerical approximation to (53) is then given by:

$S F(d)=\frac{\theta_{1}^{U}-\theta_{1}^{L}}{2} \sum_{q_{1}=1}^{Q P_{1}} w_{q_{1}}\left[\frac{\theta_{2}^{U}\left(\theta_{1}^{q_{1}}\right)-\theta_{2}^{L}\left(\theta_{1}^{q_{1}}\right)}{2}\right] \ldots$

$\sum_{q_{2}=1}^{Q P_{2}} w_{q_{2}}\left[\frac{\theta_{3}^{U}\left(\theta_{1}^{q_{1}}, \theta_{2}^{q_{1} q_{2}}\right)-\theta_{3}^{L}\left(\theta_{1}^{q_{1}}, \theta_{2}^{q_{1} q_{2}}\right)}{2}\right] \cdots$

$\sum_{q_{M-1}=1}^{Q P_{M}} w_{q_{2 M-1}}\left[\frac{\theta_{M}^{U}\left(\theta_{1}^{q_{1}}, \theta_{2}^{q_{1} q_{2}}, \cdots, \theta_{M-1}^{q_{1} q_{2} \cdots q_{M-1}}\right)-\theta_{M}^{L}\left(\theta_{1}^{q_{1}}, \theta_{2}^{q_{1} q_{2}}, \cdots, \theta_{M-1}^{q_{1} q_{2} \cdots q_{M-1}}\right)}{2}\right] \cdots$

$\sum_{q_{M}=1}^{Q P_{M}} w_{q_{M}} j\left(\theta_{1}^{q_{1}}, \theta_{2}^{q_{1} q_{2}}, \cdots, \theta_{M}^{q_{1} q_{2} \cdots q_{M}}\right)$

where $w_{q_{i}}$ are the weights corresponding to each quadrature point.

\subsection{Global optimization for the flexibility test and flexibility index problems}

The active set method in (26) and (28) developed by Grossmann \& Floudas (1987) relies on the KKT conditions to solve the inner optimization problems of the flexibility test and the flexibility index problems. If some of the model equations $\left(h_{i}(d, x, z, \theta)\right)$ or the feasibility constraints $\left(g_{j}(d, x, z, \theta)\right)$ are not convex, the KKT conditions are not sufficient for global optimality. 
Therefore, the solution obtained for the outer optimization problems might not indicate the actual flexibility of the system.

The global optimization strategy proposed by Floudas et al. (2001) for the flexibility test and the flexibility index problems is based on the principles of spatial branch and bound algorithms. Starting from the formulations obtained with the active set method (26) and (28), upper and lower bounds on the global optimum are improved by analyzing successive partitions of the space of the variables. Upper bounds on the global optimum are calculated from local solutions of the single-stage optimization problem that is obtained when the inner minimization is replaced by its KKT conditions. Lower bounds are calculated by convexification of the model equations and the feasibility constraints. Underestimators of functions $g_{j}$ and $h_{i}$ are used to relax the feasible region. The non-convex terms are replaced by their corresponding convex underestimators:

$$
\begin{aligned}
& g_{j}(d, x, z, \theta) \leq 0 \quad \Rightarrow \quad \hat{g}_{j}(d, x, z, \theta) \leq 0 \\
& h_{i}(d, x, z, \theta)=0 \quad \Rightarrow \quad \hat{h}_{i}(d, x, z, \theta)=0
\end{aligned}
$$

The flexibility test problem as stated in (32) can be expressed as follows for the convexified functions:

$$
\begin{array}{clr}
\max _{u, \theta, z, \hat{\lambda}_{j}, \hat{\mu}_{i}, \hat{s}_{j}, \hat{y}_{j}} & u & \\
\text { s.t. } & \hat{h}_{i}(d, x, z, \theta)=0 & \\
& & \\
& \hat{g}_{j}(d, x, z, \theta)+s^{j}=u \\
& \sum_{j \in J} \hat{\lambda}_{j}=1 & \\
& \sum_{i \in I} \hat{\mu}_{i} \frac{\partial \hat{h}_{i}(d, x, z, \theta)}{\partial z}+\sum_{j \in J} \hat{\lambda}_{j} \frac{\partial \hat{g}_{j}(d, x, z, \theta)}{\partial z}=0 & \\
& \sum_{i \in I} \hat{\mu}_{i} \frac{\partial \hat{h}_{i}(d, x, z, \theta)}{\partial x}+\sum_{j \in J} \hat{\lambda}_{j} \frac{\partial \hat{g}_{j}(d, x, z, \theta)}{\partial x}=0 & \\
& \hat{s}_{j} \leq U\left(1-\hat{y}_{j}\right) & j \in J \\
& \hat{\lambda}_{j} \leq \hat{y}_{j} & \\
& \hat{y}_{j}=n_{z}+1 & \\
& \theta^{L} \leq \theta \leq \theta \\
& \hat{\lambda}_{j}, \hat{s}_{j} \geq 0 ; \quad \hat{y}_{j} \in\{0,1\} & j \in J
\end{array}
$$

In order to obtain a convex formulation, further relaxation of the feasible region might be needed to eliminate non-linearities of the equality constraints, including the equality formulation of the feasibility constraints required for the active set method. If the underestimation of the model equations, feasibility constraints or the KKT conditions is non-linear, they have to be rewritten as pairs of inequalities. Eq. (60) shows the procedure for the subset of non-linear model equations.

$$
\begin{array}{ccc} 
& \hat{h}_{i^{\prime}}^{1}(d, x, z, z, \theta) \leq 0 \\
& -\hat{h}_{i^{\prime}}^{2}(d, x, z, \theta) \leq 0
\end{array}
$$


The convex relaxation of formulation (59) yields a lower bound on the flexibility test problem for any partition of the space of the variables because it implies a relaxation of the inner minimization problem. The successive refining of the partition together with the update of underestimators produces a non-decreasing sequence of lower bounds. Partitions of the space of the variables (nodes) are fathomed when the solution of the convex relaxation is greater than the best upper bound found. A similar strategy can be applied to the flexibility index problem by using a convex relaxation of formulation (28).

\subsection{Flexibility analysis of dynamic systems}

The ability of dynamic systems to maintain feasibility over ranges of uncertain parameters was studied by Dimitriadis \& Pistikopoulos (1995). They extended the flexibility test and flexibility index problems (Halemane \& Grossmann, 1983; Swaney \& Grossmann 1985) developed for steady-state models to dynamic systems that contain time-varying uncertain parameters. The main difference with the steady-state problems is given by the time-dependence of the uncertainty and the feasible region.

The dynamic flexibility problem establishes the ability of a system to maintain feasible operation throughout a finite time horizon for any possible trajectory of the uncertain parameters. If the system is found feasible for the range of uncertain parameters, the solution involves finding the control actions that guarantee feasibility in the worst trajectory of the uncertain parameters. Following the notation used for the steady-state, dynamic systems can be modeled by a set by algebraic and differential equations with the following form:

$h(d, \dot{x}(t), x(t), z(t), \theta(t), t)=0$

$x(0)=x^{0}$

The constraints that represent feasible operation are divided in two groups. Path constraints, which must be satisfied throughout the whole time horizon:

$g^{\text {path }}(d, x(t), x(t), \theta(t), t) \leq 0$

and point constraints that must be satisfied at specific instances of time:

$g_{k}^{\text {point }}\left(d, x\left(t^{k}\right), z\left(t^{k}\right), \theta\left(t^{k}\right), t^{k}\right) \leq 0$

The flexibility test problem is formulated as follows:

$$
\begin{array}{ll}
\chi(d)=\max _{\theta(t) \in T(t)} & \psi(d, \theta(t)) \\
\text { s.t. } & \psi(d, \theta(t))=\min _{z(t) \in Z(t)} \max _{j \in J, t \in[0, H]} g^{j}(d, x(t), z(t), \theta(t), t) \\
\text { s.t. } & h(d, \dot{x}(t), x(t), z(t), \theta(t), t)=0 \\
& x(0)=x^{0} \\
& T(t)=\left\{\theta(t) \mid \theta^{L}(t) \leq \theta(t) \leq \theta^{U}(t)\right\}
\end{array}
$$




$$
Z(t)=\left\{z(t) \mid z^{L}(t) \leq z(t) \leq z^{U}(t)\right\}
$$

where $J$ is the set of constraints that represent feasible operation. Just like in the steady-state case, a design $(d)$ is feasible for any trajectory of uncertain parameters if $\chi(d) \leq 0$.

Similarly, the dynamic flexibility index problem is formulated as follows:

$$
\begin{aligned}
& D F(d)=\max \delta \\
& \text { s.t. } \quad \psi \chi(d)=\max _{\theta(t) \in T(t)} \min _{z(t) \in Z(t)} \max _{j \in J, t \in[0, H]} g^{j}(d, x(t), z(t), \theta(t), t) \\
& \text { s.t. } \quad h(d, \dot{x}(t), x(t), z(t), \theta(t), t)=0 \\
& x(0)=x^{0} \\
& T(t)=\left\{\theta(t) \mid \theta^{L}(t) \leq \theta(t)\right. \\
&\left.\leq \theta^{U}(t)\right\}
\end{aligned}
$$

The dynamic flexibility index $(D F)$ quantifies the maximum range of uncertain parameters that can be tolerated throughout the time horizon while maintaining feasibility. The uncertainty ranges are measured from a nominal point $\left(\theta^{N}(t)\right)$ with the scaled deviation from the expected deviations $(\Delta \theta(t))$.

The resulting flexibility test and flexibility index formulations are two-stage semi-infinite dynamic optimization problems. Their solution can be obtained by combining a discretization scheme with the active constraint strategy developed for the steady state problems. By using orthogonal collocation on finite elements and enforcing profile continuity among elements, the dynamic flexibility test can be expressed in the following form.

$\max u$

s.t. $\quad h_{m, n}^{i}(d, x, z, \theta, t, \alpha)=0$

$$
\begin{aligned}
& g_{m, n}^{j}(d, x, z, \theta, t)+s_{m, n}^{j}-u=0 \\
& \sum_{m, n, i} \mu_{m, n}^{i} \frac{\partial h_{m, n}^{i}}{\partial x}+\sum_{m, n, j} \lambda_{m, n}^{j} \frac{\partial g_{m, n}^{j}}{\partial x}=0 \\
& \sum_{m, n, i} \mu_{m, n}^{i} \frac{\partial h_{m, n}^{i}}{\partial z}+\sum_{m, n, j} \lambda_{m, n}^{j} \frac{\partial g_{m, n}^{j}}{\partial z}=0 \\
& \sum_{m, n, i} \mu_{m, n}^{i} \frac{\partial h_{m, n}^{i}}{\partial \alpha}+\sum_{m, n, j} \lambda_{m, n}^{j} \frac{\partial g_{m, n}^{j}}{\partial \alpha}=0
\end{aligned}
$$




$$
\begin{aligned}
& \sum_{m, n, j} \lambda_{m, n}^{j}=1 \\
& \sum_{m, n, j} y_{m, n}^{j}=n_{z}+1 \\
& \lambda_{m, n}^{j}-y_{m, n}^{j} \leq 0 \\
& s_{m, n}^{j}-U\left(1-y_{m, n}^{j}\right) \leq 0 \\
& \theta^{L} \leq \theta \leq \theta^{U} \\
& y_{m, n}^{j} \in\{0,1\} \quad \lambda_{m, n}^{j}, s_{m, n}^{j} \geq 0 \\
& m \in M \quad n \in N \quad i \in I \quad j \in J
\end{aligned}
$$

where $M$ is the set of finite elements, $N$ the set of collocation points, $I$ the set of model equations, and $J$ the set of feasibility constraints. The location of the discrete-time elements is indicated by index $t$ and the collocation points are denoted by $\alpha$. The Lagrange multipliers of the model and the feasibility constraints are $\mu$ and $\lambda$, respectively. Slack variables $\left(s_{m, n}^{j}\right)$ are used in the same way as in the steady-state model; the model selects the active constraints with binary variables $y_{m, n}^{j}$.

The dynamic flexibility index problem can also be transformed to a mixed-integer formulation following the same principles.

$$
\begin{array}{ll}
\min & \delta \\
\text { s.t. } & h_{m, n}^{i}(d, x, z, \theta, t, \alpha)=0 \\
& g_{m, n}^{j}(d, x, z, \theta, t)+s_{m, n}^{j}=0 \\
& \sum_{m, n, i} \mu_{m, n}^{i} \frac{\partial h_{m, n}^{i}}{\partial x}+\sum_{m, n, j} \lambda_{m, n}^{j} \frac{\partial g_{m, n}^{j}}{\partial x}=0 \\
& \sum_{m, n, i} \mu_{m, n}^{i} \frac{\partial h_{m, n}^{i}}{\partial z}+\sum_{m, n, j} \lambda_{m, n}^{j} \frac{\partial g_{m, n}^{j}}{\partial z}=0 \\
& \sum_{m, n, i} \mu_{m, n}^{i} \frac{\partial h_{m, n}^{i}}{\partial \alpha}+\sum_{m, n, j} \lambda_{m, n}^{j} \frac{\partial g_{m, n}^{j}}{\partial \alpha}=0 \\
& \sum_{m, n, j} \lambda_{m, n}^{j}=1 \\
& \sum_{m, n, j} y_{m, n}^{j}=n_{z}+1
\end{array}
$$




$$
\begin{aligned}
& \lambda_{m, n}^{j}-y_{m, n}^{j} \leq 0 \\
& s_{m, n}^{j}-U\left(1-y_{m, n}^{j}\right) \leq 0 \\
& \theta^{N}-\delta \Delta \theta^{-} \leq \theta \leq \theta^{U}+\delta \Delta \theta^{+} \\
& y_{m, n}^{j} \in\{0,1\} \quad \lambda_{m, n}^{j}, s_{m, n}^{j} \geq 0 \quad \delta \geq 0 \\
& m \in M \quad n \in N \quad i \in I \quad j \in J
\end{aligned}
$$

These formulations can accommodate additional constraints that impose restrictions on the performance of the control variables. By doing so, the feasibility of systems with realistic control strategies can be analyzed.

\section{Conclusions}

This paper has given a historical perspective and an overview of the pioneering work that Manfred Morari developed in the area of resiliency, a major component of process operability. Motivated by unique counter-intuitive process examples, a review has been presented of the early mathematical formulations and solution methods developed by Grossmann and co-workers for addressing Static Resiliency (Flexibility) problems, which in fact are conceptually related to the area of robust optimization (Ben-Tal et al., 2009) for the case of design optimization subject to flexibility constraints. We also give a brief overview of some of the seminal ideas by Morari and co-workers in the area of Dynamic Resiliency. Finally, we have reviewed some of the recent developments that have taken place since that early work took place, which clearly indicates that on the one hand there has been significant progress in this area, and on the other hand it also indicates that there are still major challenges that remain to be addressed in this area, including their application in industry. 


\section{References}

Banerjee, I. \& Ierapetritou, M. G. (2005). Feasibility Evaluation of Nonconvex Systems Using Shape Reconstruction Techniques. Industrial and Engineering Chemistry Research, 44(10), 3638-3647.

Ben-Tal, A., El Ghaoui, L. \& Nemirovski, A. (2009). Robust Optimization. Princeton, NJ: Princeton University Press

Biegler, L. T., Grossmann, I. E. \& Westerberg, A. W. (1997). Systematic methods for chemical process design. Old Tappan, NJ: Prentice Hall.

Birge, J. R. \& Louveaux, F. (2011). Introduction to Stochastic Programming (2nd ed). New York, NY: Springer.

Boukouvala, F. \& Ierapetritou, M. G. (2012). Feasibility Analysis of Black-Box Processes Using an Adaptive Sampling Kriging-based Method. Computers and Chemical Engineering, 36(10), 358-368.

Dimitriades, V. D. \& Pistikopoulos, E. N. (1995). Flexibility Analysis of Dynamic Systems. Industrial and Engineering Chemistry Research, 34, 4451.

Floudas, C. A. \& Grossmann, I. E. (1987). Synthesis of Flexible Heat Exchanger Networks with Uncertain Flowrates and Temperatures. Computers and Chemical Engineering, 11, 319.

Floudas, C. A., Gümüs, Z. H., \& Ierapetritou, M. G. (2001). Global Optimization in Design under Uncertainty: Feasibility Test and Flexibility Index Problems. Industrial and Engineering Chemistry Research, 40, 4267-4282.

Goyal, V. \& Ierapetritou, M. G. (2002). Determination of Operability Limits Using Simplicial Approximation. AIChE Journal, 48(12), 2902-2909.

Grossmann, I. E. \& Floudas, C. A. (1987). Active Constraint Strategy for Flexibility Analysis in Chemical Processes. Computers and Chemical Engineering, 11, 675.

Grossmann, I. E., Halemane, K. P. \& Swaney, R. E. (1983). Optimization Strategies for Flexible Chemical Processes. Computers and Chemical Engineering, 7, 439.

Grossmann, I. E. \& Morari, M. (1984). Operability, Resiliency and Flexibility-Process Design Objectives for a Changing World. Proc. In A. W. Westerberg \& H. H. Chien (Eds.), Prod. $2^{\text {nd }}$ International Conference on Foundations Computer Aided Process Design, CACHE, vol. 937.

Grossmann, I. E. \& Sargent, R. W. H. (1978). Optimum Design of Chemical Plants with Uncertain Parameters. AIChE Journal, 24, 1021. 
Grossmann, I. E. \& Sargent, R. W. H. (1979). Optimum Design of Multipurpose Chemical Plants. Ind. Eng. Chem. Process Des. Development 18, 343.

Grossmann, I. E. \& Straub, D. A. (1991). Recent Developments in the Evaluation and Optimization of Flexible Chemical Processes. In L. Puigjaner \& A. Espuna (Eds.), Proceedings of COPE-91 (pp. 49-59).

Halemane, K. P. \& Grossmann, I. E. (1983). Optimal Process Design under Uncertainty. AIChE Journal, 29, 425.

Johns, W. R., Marketos, G. \& Rippin, D. W. T. (1976). The Optimal Design of Chemical Plant to Meet Time-varying Demands in the Presence of Technical and Commercial Uncertainty. Design Congress 76, F1.

Kabatek, U. \& Swaney, R. E. (1992). Worst-Case Identification in Structured Process Systems. Computers and Chemical Engineering, 16, 1063-1072.

Kreisselmeier, G. \& Steinhauser, R. (1979). Systematic Control Design by Optimizing a Vector Performance Index. In Proceedings of IFAC Computer Aided Design of Control Systems.

Lennhoff, A. M. \& Morari, M. (1982). Design of Resilient Processing Plants. I. Process Design under Consideration of Dynamic Aspects. Chemical Engineering Science, 37, 245.

Malik, R. K. \& Hughes, R. R. (1979). Optimal Design of Flexible Chemical Processes. Computers and Chemical Engineering, 3, 473.

Morari, M. (1983). A general framework for the assessment of dynamic resilience. Chemical Engineering Science, 38(11), 1881-1891.

Pistikopoulos, E. N. \& Grossmann, I. E. (1988). Optimal Retrofit Design for Improving Process Flexibility in Linear Systems. Computers and Chemical Engineering, 12, 719.

Pistikopoulos, E. N. \& Grossmann, I. E. (1989). Optimal Retrofit Design for Improving Process Flexibility in Nonlinear Systems- I Fixed Degree of Flexibility. Computers and Chemical Engineering, 13, 1003.

Pistikopoulos, E. N. \& Mazzuchi, T. A. (1990). A Novel Flexibility Analysis Approach for Processes with Stochastic Parameters. Computers and Chemical Engineering, 14(9), 991-1010.

Rooney, W. C. \& Biegler, L. T. (2001). Design for Model Parameter Uncertainty using Nonlinear Confidence Regions. AIChE Journal, 47(8), 1794-1804.

Rooney, W. C. and Biegler, L. T. (2004). Optimal Process Design with Model Parameter Uncertainty and Process Variability. AIChE Journal, 49(2), 438-449. 
Saboo, A. K. \& Morari, M. (1984). Design of Resilient Processing Plants - IV: Some New Results on Heat Exchanger Network Synthesis. Chemical Engineering Science, 39, 579-592.

Straub, D. A. \& Grossmann, I. E. (1990). Integrated Statistical Metric of Flexibility for Systems with Discrete State and Continuous Parameter Uncertainties. Computers and Chemical Engineering, 14, 967.

Swaney, R. E. \& Grossmann, I. E. (1985a). An Index for Operational Flexibility in Chemical Process Design. Part 1 Formulation and Theory. AIChE Journal, 31, 621.

Swaney, R. E. \& Grossmann, I. E. (1985b). An Index for Operational Flexibility in Chemical Process Design. Part 2 Computational Algorithms. AIChE Journal, 31, 631.

Varvarezos, D. K., Grossmann, I. E. \& Biegler, L. T. (1992). An Outer Approximation Method for Multiperiod Design Optimization. Industrial and Engineering Chemistry Research, 31, 1466-1477.

Varvarezos, D. K., Grossmann, I. E. \& Biegler, L. T. (1994). Multiperiod Design Optimization with SQP Decomposition. Computers and Chemical Engineering, 18, 579. 